\title{
Real time structural modification of epitaxial FePt thin films under x-ray rapid thermal annealing using undulator radiation
}

\author{
J. R. Skuza ${ }^{a)}$ and R. A. Lukaszew ${ }^{\text {b) }}$ \\ Department of Physics and Astronomy, The University of Toledo, Toledo, Ohio 43606 \\ E. M. Dufresne and D. A. Walko \\ Advanced Photon Source, Argonne National Laboratory, Argonne, Illinois 60439 \\ C. Clavero and A. Cebollada \\ Instituto de Microelectrónica de Madrid-IMM (CNM-CSIC), Isaac Newton 8-PTM, \\ 28760 Tres Cantos, Madrid, Spain \\ C. N. Cionca and R. Clarke \\ Department of Physics, University of Michigan, Ann Arbor, Michigan 48109
}

(Received 20 March 2007; accepted 23 May 2007; published online 18 June 2007)

\begin{abstract}
Modification of chemical order in epitaxial FePt binary alloy thin films deposited on $\mathrm{MgO}$ (100) substrates was induced and investigated in real time using x-ray rapid thermal annealing (XRTA). This is possible because synchrotron undulator radiation has sufficient power density to induce significant structural modifications in thin films and its energy can be tuned to optimize absorption in the sample. A monochromatic portion of the pink beam diffracted from the epitaxial FePt sample was used to probe microstructure evolution in real time and significant changes in chemical order were observed. In particular, the relative amount of $L 1_{0}$ phase remained practically unchanged whereas the amount of $L 1_{2}$ phase was significantly decreased in the FePt thin film sample during XRTA. (C) 2007 American Institute of Physics. [DOI: 10.1063/1.2749426]
\end{abstract}

Thermal treatments applied to thin film alloy samples after growth can induce phase transformations which are controlled by nucleation and growth kinetics and driven by diffusion mechanisms. ${ }^{1}$ Previous work suggested that microstructure changes during these phase transformations depend not only on the annealing temperature but also on the heating rate used during the heating cycle. ${ }^{2}$ In the case of multilayered FePt films for example, the diffusion lengths and time scales for these processes have been shown to follow two different regimes based on the annealing temperature. ${ }^{3}$ Thus, we have explored the use of a variation of rapid thermal annealing (RTA) to modify and control the microstructure in epitaxial FePt thin films while monitoring these changes in real time.

FePt binary alloys comprise a rich system exhibiting several chemically ordered phases based on the stoichiometry of the alloy. ${ }^{4,5}$ Among them are the $L 1_{0}$ and $L 1_{2}$ chemically ordered phases that form in the vicinity of $1: 1$ and $3: 1$ (or 1:3) Fe:Pt stoichiometry, respectively. The $L 1_{0}$ structure is characterized by intercalating $\mathrm{Fe}$ and $\mathrm{Pt}$ atomic layers along a cubic stacking direction, which as a consequence suffers a tetragonal distortion (i.e., the lattice parameter along the stacking direction is contracted). ${ }^{6}$ The $L 1_{2}$ structure is distinguished by one of the atomic species occupying the corner of a cubic lattice and the other occupying the three face centers. ${ }^{6}$ Suitable combinations of the $L 1_{0}$ and $L 1_{2}$ phases have been proposed for the next generation magnetic recording media, where the strong perpendicular anisotropy of the $L 1_{0}$ phase can be combined with the in-plane aniso-

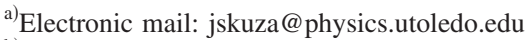

${ }^{b)}$ Author to whom correspondence should be addressed; electronic mail: alukasz@physics.utoledo.edu
}

tropy of the $L 1_{2}$ phase to yield media with tilted magnetization. ${ }^{7-9}$

The microstructure changes occurring during these phase transformations upon annealing can be monitored using structure-sensitive techniques such as $\mathrm{x}$-ray diffraction (XRD). ${ }^{6}$ Here, we present an application of x-ray undulator radiation to simultaneously modify and probe structural changes that occur in epitaxial FePt thin films under x-ray rapid thermal annealing (XRTA) in real time.

Epitaxial FePt thin films were sputter-deposited in an ultrahigh vacuum deposition system (base pressure of $\sim 2$ $\times 10^{-9}$ Torr) using high purity $(99.99 \%) \mathrm{Fe}$ and Pt targets. $\mathrm{MgO}$ buffer layers $(\sim 100 \AA)$ were deposited onto the singlecrystal $\mathrm{MgO}(100)$ substrates at $450{ }^{\circ} \mathrm{C}$ by laser ablation. High-purity Ar was used during the cosputtering of $\mathrm{Fe}$ and Pt. All samples studied had a nominal film thickness of $\sim 300 \AA$ after $30 \mathrm{~min}$ of growth. The composition of the alloy was adjusted to be slightly Fe rich to explore the evolution of more than one of the ordered phases (i.e., $L 1_{0}$ and $L 1_{2}$ ) and thus evaluate the interplay between out-of-plane and in-plane magnetic anisotropies upon annealing the thin film samples. The deposition temperature was varied between 450 and $600{ }^{\circ} \mathrm{C}$ to further tailor the degree of partial chemical order in the as-grown samples. We probed the crystallographic texture of the as-grown films using $\mathrm{Cu} K \alpha \mathrm{XRD}$. Film microstructure was studied by a JEOL $2010 \mathrm{~F}$ (S) transmission electron microscope (TEM) operating at $200 \mathrm{kV}$.

Annealing studies on the epitaxial FePt thin films were carried out ex situ after growth. Here, the XRTA method exploits the properties of $\mathrm{x}$-ray undulator radiation to induce rapid thermal annealing, while probing structural changes in films in real time. The XRTA experiment applied to epitaxial FePt thin films was performed at the Advanced Photon Source at Argonne National Laboratory utilizing the Sector 7 


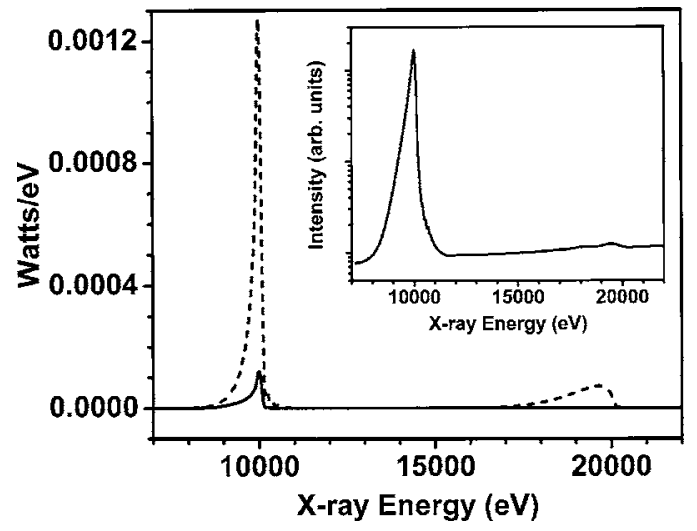

FIG. 1. Theoretical spectral power of the white (dashed trace) and pink (solid trace) beams up to a photon energy of $22 \mathrm{keV}$. The integrated power delivered by the pink beam is $\sim 46.2 \mathrm{~mW}$ compared to $\sim 700 \mathrm{~mW}$ delivered by the white beam. The inset shows a specular $(\theta-2 \theta)$ XRD scan of a $\mathrm{Si}$ (111) reflection from the analyzer crystal. The undulator fundamental energy was set at $10 \mathrm{keV}$.

beamline. The white beam (i.e., the full spectral output of the synchrotron) was produced by an undulator tuned to produce a spectrum with the peak of the first harmonic at $10 \mathrm{keV}$. The beam was collimated by slits placed $26.5 \mathrm{~m}$ from the source and set to an opening of $0.1 \times 0.1 \mathrm{~mm}^{2}$ then reflected from a water-cooled flat $\mathrm{Rh}$ mirror at a vertical grazing angle of $0.415^{\circ}$. The mirror attenuated the higher harmonics in the white beam and thus produced the desired pink beam (i.e., a spectral peak with $\sim 2 \%$ energy bandwidth). The full width at half maximum of the pink beam footprint on the sample was measured to be $0.34 \times 0.16 \mathrm{~mm}^{2}$ in the horizontal and vertical directions, respectively. In order to probe the microstructure evolution of the sample during the XRTA process, a monochromatic portion of the diffracted pink beam was selected by an analyzer crystal. A Si (111) crystal was mounted on the analyzer stage of the diffractometer's detection arm, providing a means of high-resolution, real time detection of the sample's diffraction peaks.

An important aspect of XRTA is that power absorption on the sample can be optimized using the tunability of the beam energy. Thus, for the present case the beam energy was set at $10 \mathrm{keV}$, which is above the Fe $K$ edge $(7.1 \mathrm{keV})$. We note that although the full power produced by the undulator at $10 \mathrm{keV}$, with $100 \mathrm{~mA}$ current in the accelerator, is $\sim 608 \mathrm{~W}$, collimation and attenuation significantly reduced the power delivered to the sample. This power can be calculated by integrating the area under the theoretical spectral curve in Fig. 1. Thus, the total power delivered by the white beam was $\sim 700 \mathrm{~mW}$, and it was reduced to $\sim 46.2 \mathrm{~mW}$ in the reflected pink beam incident on the sample. The power absorbed by the FePt thin film and $\mathrm{MgO}$ substrate was calculated to be $\sim 0.8$ and $\sim 45.4 \mathrm{~mW}$, respectively, using the XPOWER module of the software package XOP. ${ }^{10}$ The inset in Fig. 1 shows a specular $(\theta-2 \theta)$ XRD scan of a Si (111) reflection from the analyzer crystal with the undulator fundamental energy set at $10 \mathrm{keV}$.

Noting that the actual heat transfer during XRTA depends on the experimental setup, we observed that the power delivered to the FePt thin film sample in the present experiment was sufficient to induce significant structural modifications. The XRD scans were obtained in real time in $\theta-2 \theta$ geometry using a six-circle Huber diffractometer located $37.5 \mathrm{~m}$ from the source. Figure 2 compares the intensities of Downloaded 16 Jun 2009 to 161.111.235.43. Redistribution subject
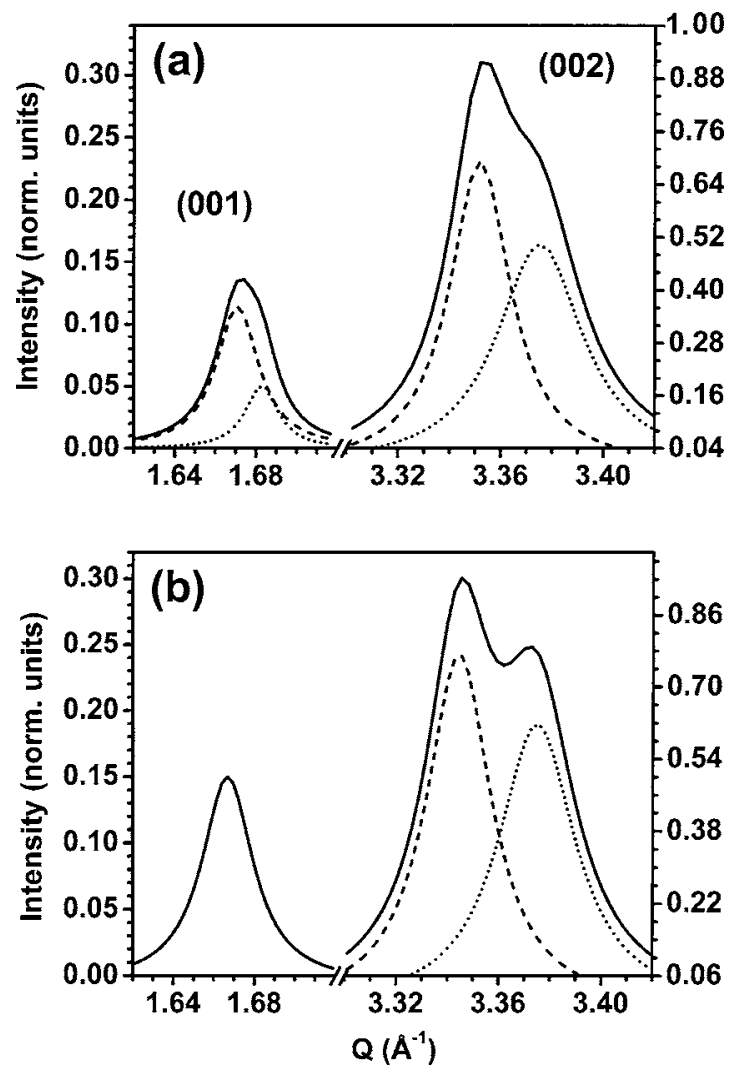

FIG. 2. Specular $(\theta-2 \theta)$ XRD scans of the superstructure (001) and fundamental (002) reflections of an epitaxial FePt thin film. (a) RT scan of the as-grown film and (b) scan of the same film during XRTA. The noticeable splitting of the fundamental reflections during annealing is clear evidence of changes in the microstructure. The presence of superstructure peaks reflects some degree of chemical order. The data (solid lines) are fit to peaks corresponding to the $L 1_{0}$ and $L 1_{2}$ phases (dashed and dotted lines, respectively).

the FePt reflections that are perpendicular to the film plane [i.e., the superstructure (001) and fundamental (002) reflections] for the as-grown films [Fig. 2(a)] to those of the same films under XRTA for 10 min (Refs. 11 and 12) [Fig. 2(b)]. The observed (001) and (002) FePt reflections are ascribed to the various phases present in the thin film sample, including chemically ordered $L 1_{0}$ and $L 1_{2}$ structures. Two fundamental (002) reflections were fitted in the $Q$ region of $3.30-3.42 \AA^{-1}$, where $Q$ is the momentum transfer perpendicular to the film plane. The two corresponding (001) superstructure reflections were fitted in the $Q$ region of $1.62-1.71 \AA^{-1}$. Thus, from the fits for the as-grown film, we infer that both $L 1_{0}$ and $L 1_{2}$ chemically ordered phases are present with lattice parameters of 3.76 and $3.72 \AA$, respectively. Based on the shifts of the $L 1_{0}$ diffraction peaks during XRTA, we estimate a film temperature of $\sim 500^{\circ} \mathrm{C}$ under XRTA. Furthermore, the $L 1_{2}$ diffraction peaks do not shift due to the high sensitivity of the thermal expansion coefficient to the Invar effect, ${ }^{13}$ thus complicating an accurate temperature measurement.

The structural modifications occurring in the film under XRTA can be qualitatively observed by the splitting of the $L 1_{0}$ and $L 1_{2}$ fundamental reflections. Quantitative analysis of the structural changes can be carried out by integrating the FePt $(00 L)$ peak areas to calculate $S$, a one-dimensional long-range chemical ordering parameter, before and during annealing treatment. ${ }^{6,14} S$ can be expressed in terms of the integrated areas $(A)$ of the diffraction peaks, Lorentzto AIP license or copyright; see http://apl.aip.org/apl/copyright.jsp 


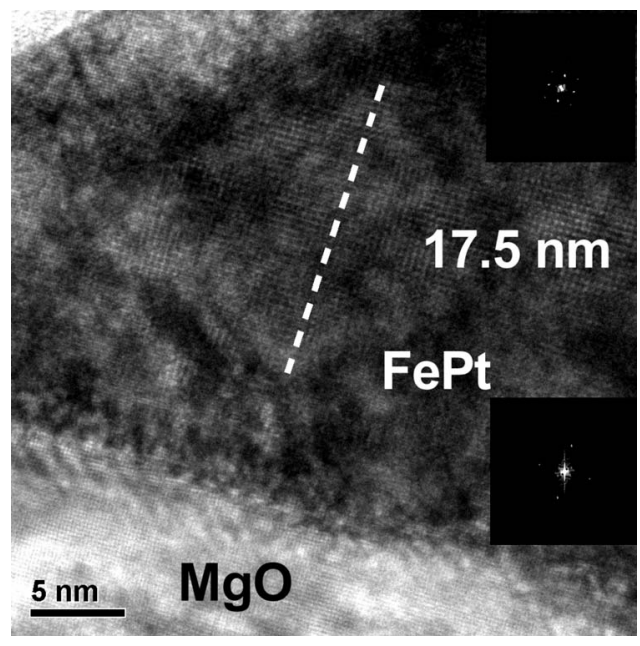

FIG. 3. High resolution (HRTEM) image of an as-grown epitaxial FePt thin film where a chemically ordered $\left(L 1_{2}\right)$ grain near the film/MgO buffer layer interface can be observed. The average size of $L 1_{2}$ ordered regions determined from XRD scans is consistent with the dimension of the $L 1_{2}$ ordered region in this image. The top inset shows the FFT of this ordered grain, while the low inset shows the FFT of a disordered region. Thus, we notice the doubled periodicity in two orthogonal directions of the ordered region with respect to the disordered one.

polarization (LP) factors for a single crystal, ${ }^{14}$ and the square magnitude of the structure factors ${ }^{6,14,15}$ calculated for the fully ordered crystal $\left(\mathrm{FF}^{*}\right)$, such that

$$
\frac{A_{001}}{A_{002}}=\frac{\mathrm{LP}_{001}}{\mathrm{LP}_{002}} \frac{\left.\left(\mathrm{FF}^{*}\right)_{001}\right)_{002}}{\left(\mathrm{FF}^{*}\right.}
$$

where $S$ is intrinsic of $\left(\mathrm{FF}^{*}\right)_{001}$.

Using Eq. (1), we calculate $S=0.40$ for the $L 1_{2}$ phase in the as-grown film and $S=0$ for this phase in the film under XRTA. This is due to the disappearance of the superstructure peak and is interpreted as Fe and Pt atoms randomly changing sites from occupying "correct" sites to "incorrect" ones in the cubic lattice without altering the stoichiometry of this phase. On the other hand, we find that $S=0.38$ for the $L 1_{0}$ phase in the as-grown film and $S=0.41$ for this phase in the film under XRTA. Thus, we conclude that the relative amount of the $L 1_{0}$ phase has not significantly changed, while the amount of $L 1_{2}$ phase present in the sample was substantially decreased under XRTA. This observation does not preclude changes in grain size distribution upon annealing, considering that diffusion mechanisms alter smaller grains more rapidly than the larger ones. ${ }^{16}$

Figure 3 shows a high resolution transmission electron microscopy (HRTEM) image of an as-grown epitaxial FePt thin film with a fully ordered grain near the $\mathrm{MgO}$ buffer layer. Fast Fourier transform (FFT) analysis of the image of this grain (top inset) indicates two orthogonal directions of doubled periodicity corresponding to the $L 1_{2}$ ordered phase. Although, this doubled periodicity could be interpreted as orthogonal grain boundaries in the $L 1_{0}$ phase, there is no diffraction evidence of this in the XRD scans. The size of this $L 1_{2}$ ordered grain is $\sim 175 \AA$ and agrees well with values obtained by applying the Scherrer equation ${ }^{14}$ to the measured peak widths in the XRD scans. The average size thus determined for the $L 1_{2}$ regions is $200 \pm 15 \AA$ for the asgrown film and it is $175 \pm 15 \AA$ for the resulting disordered grains under XRTA, showing that chemical disorder was dominant over the grain size reduction during XRTA.

In summary, our experiments have demonstrated that undulator radiation is a very powerful tool for real time studies of microstructure changes in thin film alloy samples under rapid thermal annealing. The XRTA technique proved particularly useful to study technologically important binary alloy systems such as FePt that exhibit temperature-induced phase transformations leading to microstructure changes. Furthermore, undulator radiation can be tuned to the absorption edge of a specific element in the material to optimize heat absorption. For the case studied, significant modification of chemical order was achieved in epitaxial FePt thin films under XRTA. Particularly, the amount of $L 1_{2}$ phase was significantly reduced as compared to relatively no change in the amount of $L 1_{0}$ phase. This reduction of the $L 1_{2}$ phase possibly implies a reduction of the in-plane magnetic anisotropy, which will be reported on in the future.

This work was performed in part at beamline 7-ID. Use of the Advanced Photon Source was supported by the U.S. Department of Energy, Office of Science, Office of Basic Energy Sciences, under Contract No. DE-AC02-06CH11357. This work was partially supported by DOE Grant DE-FG0206ER46273, NSF-DMR (Grant No. 0355171), the American Chemical Society (PRF Grant No. 41319-AC), the Research Corporation Cottrell Scholar award, the Spanish Ministerio of Educación y Ciencia, and the FPI Program.

${ }^{1}$ R. Pascual, M. Sayer, C. V. R. Vasant Kumar, and L. Zou, J. Appl. Phys. 70, 2348 (1991)

${ }^{2}$ R. Pascual, M. Sayer, A. Lo, S. Herbert, L. C. Rolim, and N. Townley, J. Appl. Phys. 79, 493 (1996).

${ }^{3}$ N. Zotov, J. Feydt, A. Savan, and A. Ludwig, J. Appl. Phys. 100, 073517 (2006).

${ }^{4}$ R. Hultgren, P. A. Desai, D. T. Hawkings, M. Gleiser, and K. K. Kelly, Selected Values of Thermodynamic Properties of Binary Alloys (American Society of Metals, Metals Park, OH, 1973), p. 863.

${ }^{5}$ G. Bozzolo, R. A. Lukaszew, and J. E. Garces, Appl. Phys. Lett. 88, 011915 (2006).

${ }^{6}$ A. Cebollada, R. F. C. Farrow, and M. F. Toney, in Magnetic Nanostructures, edited by H. S. Nalwa (American Scientific, Stevenson Ranch, CA, 2002), pp. 94-100.

${ }^{7}$ Y. Y. Zou, J. P. Wang, C. H. Lee, and T. C. Chong, Appl. Phys. Lett. 82, 2473 (2003).

${ }^{8}$ K. Z. Gao and H. N. Bertram, IEEE Trans. Magn. 39, 704 (2003).

${ }^{9}$ A. K. Singh, J. Yin, H. Y. Y. Ko, and T. Suzuki, J. Appl. Phys. 99, 08 E704 (2006).

${ }^{10}$ M. Sanchez del Rio and R. J. Dejus, Proc. SPIE 3448, 340 (1998).

${ }^{11}$ No changes in the microstructure were observed for an additional $20 \mathrm{~min}$ of XRTA.

${ }^{12}$ The time evolution of the XRD scans of the sample under XRTA is not shown for clarity.

${ }^{13}$ E. F. Wassermann, in Ferromagnetic Materials, edited by K. H. J. Buschow and E. P. Wohlfarth (North-Holland, Amsterdam, 1990), Vol. 5, p. 237.

${ }^{14}$ B. E. Warren, X-ray Diffraction (Addison-Wesley, Reading, MA, 1969).

${ }^{15}$ A. Cebollada, D. Weller, J. Sticht, G. R. Harp, R. F. C. Farrow, R. F. Marks, R. Savoy, and J. C. Scott, Phys. Rev. B 50, 3419 (1994).

${ }^{16}$ T. Muller, K.-H. Heinig, and W. Moller, Appl. Phys. Lett. 81, 3049 (2002). 